\title{
Dual-band polarization-independent metamaterial absorber for radar stealth technology
}

\author{
Yao Ma ${ }^{1, \text { a }}$, Lu Feng ${ }^{2, b}$, Liu Jun ${ }^{1, ~ c}$, Liyan Zhu ${ }^{1}$ \\ ${ }^{1}$ National Key Laboratory on Electromagnetic Environmental Effects and Electro-optical \\ Engineering, PLA University of Science and Technology, Nanjing 210007, China \\ ${ }^{2}$ Jiangsu Regulatory Bureau of Nuclear and Radiation Safety, Nanjing 210019, China \\ aemail:mayao_84@aliyun.com, bemail:fenglu76@163.com, ${ }^{\mathrm{c} e m a i l}:$ nanjnliujn@163.com
}

Keywords: metamaterial absorber(MMA); dual-band; polarization-independent

\begin{abstract}
The design, simulation and analysis of a dual-band metamaterial absorber(MMA) working at the microwave frequencies are presented. The single unit cell consists of two nested closed hexagon ring resonators and a metallic background plane, separated by a dielectric layer with only 0.7 -mmthickness. The simulation results show that the absorber has two distinctive absorption peaks located at frequencies $8.1 \mathrm{GHz}$ and $10.7 \mathrm{GHz}$ with absorption rates of 0.95 and 0.94 , respectively. In addition, due to the symmetrical structure, the designed absorber is polarization insensitive.
\end{abstract}

\section{Introduction}

Metamaterial is a macroscopic composite of periodic or non-periodic structure, whose function is due to both the cellular architecture and the chemical composition [1].The main advantage of metamaterial is that new materials which are unavailable in nature can be created by using metamaterial structures. Due to its unusual features, metamaterial has a lot of applications both in civil and military domains. Metamaterial absorber (MMA) is one of the innovative ideas which can be used to reduce radar cross section (RCS) in radar stealth technology. In 2008, Landy et al. proposed the concept of perfect MMA in the GHz regime, in which the structure was composed of electric resonator and cut wire, and an absorption of 96\% was achieved [2]. However, most of the existing MMAs are single-band and polarization-sensitive with narrow accepted angels. Recently, many efforts have been made to achieve multiband, polarization-independent and wide incident angle absorption [3-7].

In this paper, we propose a simple and efficient approach to achieve a dual-band and polarization-independent absorber in the $\mathrm{GHz}$ regime. The single unit cell consists of two nested closed hexagon ring resonators and a metallic background plane, separated by a very thin dielectric layer. The absorber exhibits near perfect absorption at two resonant frequencies for both transverse electric(TE) and transverse magnetic(TM) polarization.

\section{Design}

The designed unit cell of dual-band absorber is shown in Fig. 1(a) is the front view and (b) is the side view. The MMA consists of three layers. The top layer is an array of two nested closed hexagon ring resonators made of copper, which is responsible for the electric resonance. The bottom layer is a copper plane, which is responsible for the magnetic resonance by exciting antiparallel surface current to that of the top layer. Two such layers are separated by a dielectric substrate. 


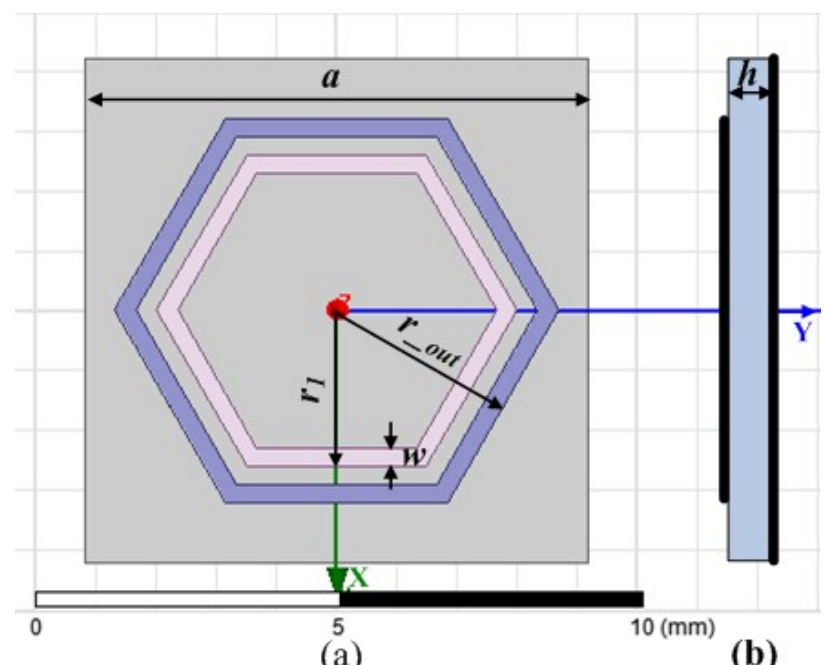

Fig. 1. (a) The top view and (b) the side view of the structure of the dual-band MMA unit cell.

We use the commercial software HFSS to make simulation and optimization of the MMA. Master and Slave Boundaries are set in the $\mathrm{x}$ and $\mathrm{y}$ directions to simulate the periodic structure, and Floquet Ports are defined in the $\mathrm{z}$ direction as the excitation. The metal material is copper with electric conductivity $5.8 \times 107 \mathrm{~S} / \mathrm{m}$ and thickness $0.018 \mathrm{~mm}$. The dielectric substrate is FR4 with relative permittivity 4.4 and dielectric loss tangent 0.02 .

The absorption is defined as $A=1-R-T$, in which $R=\left|S_{11}\right|^{2}$ represents reflection and $T=\left|S_{21}\right|^{2}$ represents transmission. Due to the metal ground plane, the transmission is zero, so the absorption is calculated as $A=1-\left|S_{11}\right|^{2}$. Through optimizing the geometry of the resonators and thickness of the substrate, we can minimize the reflection to maximize the absorption.

Fig. 2 shows how reflection coefficient S11 varies with $\mathrm{r}$ _out, from which we observe that S11 reaches minimum points at two resonance frequencies. Adjusting r_out will only affect the location of the lower resonance frequency, while the location of the higher resonance frequency is nearly unchanged. That's because each closed hexagon ring is responsible for one resonance. The inside ring response for the higher resonance frequency, while the outside ring response for the lower resonance frequency. The bigger the ring is the lower the resonance frequency is.

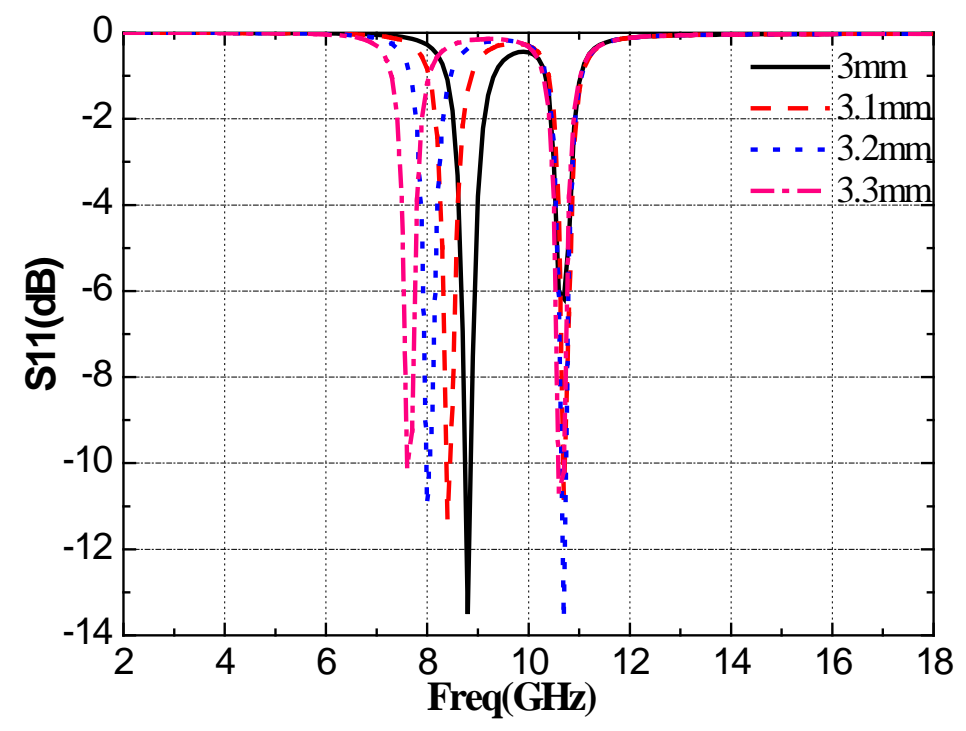

Fig. 2 Reflection coefficient S11 at various r_out. 
Fig. 3 and Fig. 4 shows how reflection coefficient S11 change with substrate length a and thickness h. As we can see change a and h will not obviously change the location of resonance frequency, but will affect the resonant strength, namely the minimum value of S11. This is because as we mentioned before, the top layer is mainly responsible for electric resonance, while the bottom layer is responsible for the magnetic resonance. Thus change a and $\mathrm{h}$ in fact affect the magnetic resonance and we need to adjust them to optimize the magnetic resonance.

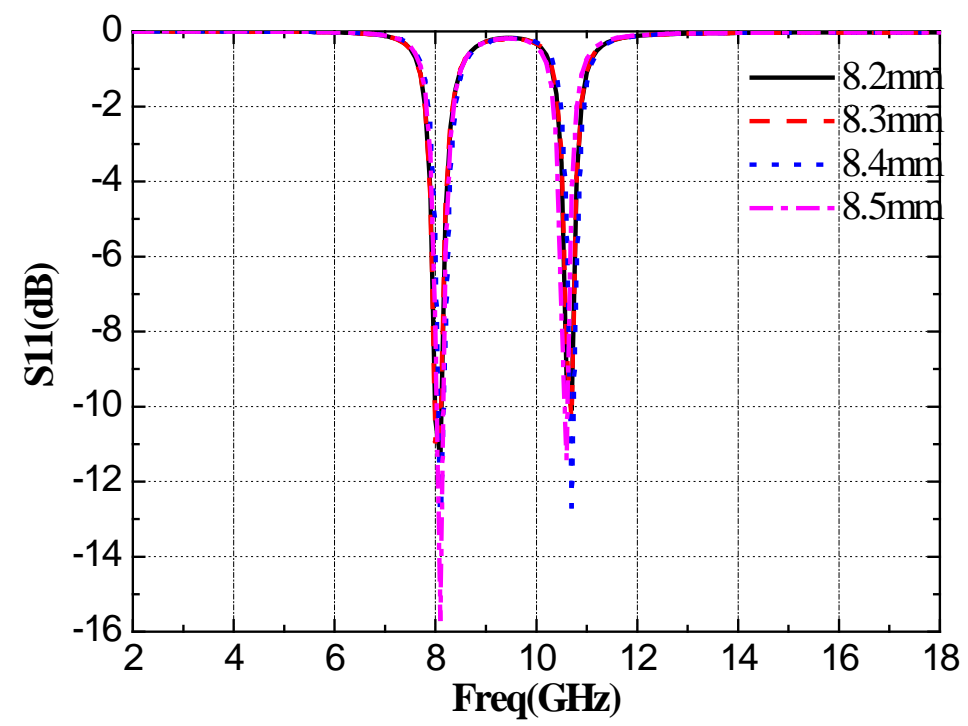

Fig. 3 Reflection coefficient S11 at various a.

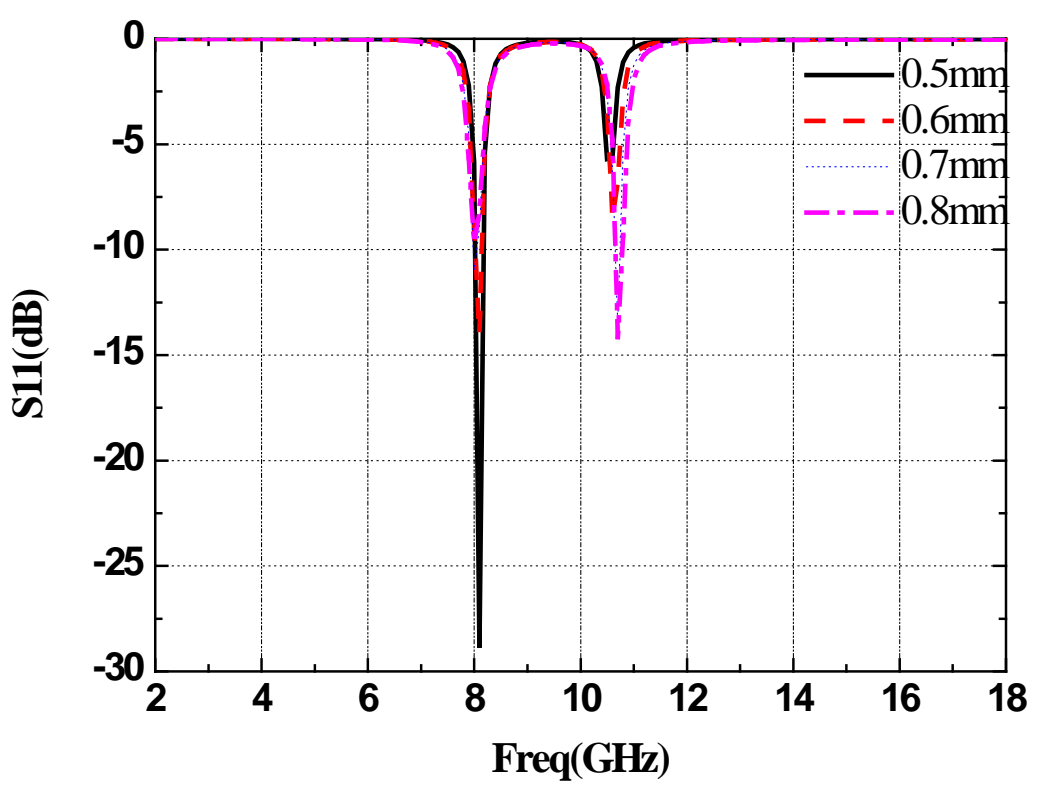

Fig. 4 Reflection coefficient S11 at various h.

Based on the research above, the final optimized dimensions of the MMA unit cell is given by:a=8.4mm, $r 1=2.6 \mathrm{~mm}, w=0.3 \mathrm{~mm}, \mathrm{r}_{-}$out $=3.2 \mathrm{~mm}, \mathrm{~h}=0.7 \mathrm{~mm}$. Fig.5 and Fig. 6 show reflection coefficient S11 and absorption rate for both transverse electric (TE) and transverse magnetic (TM) polarization. 


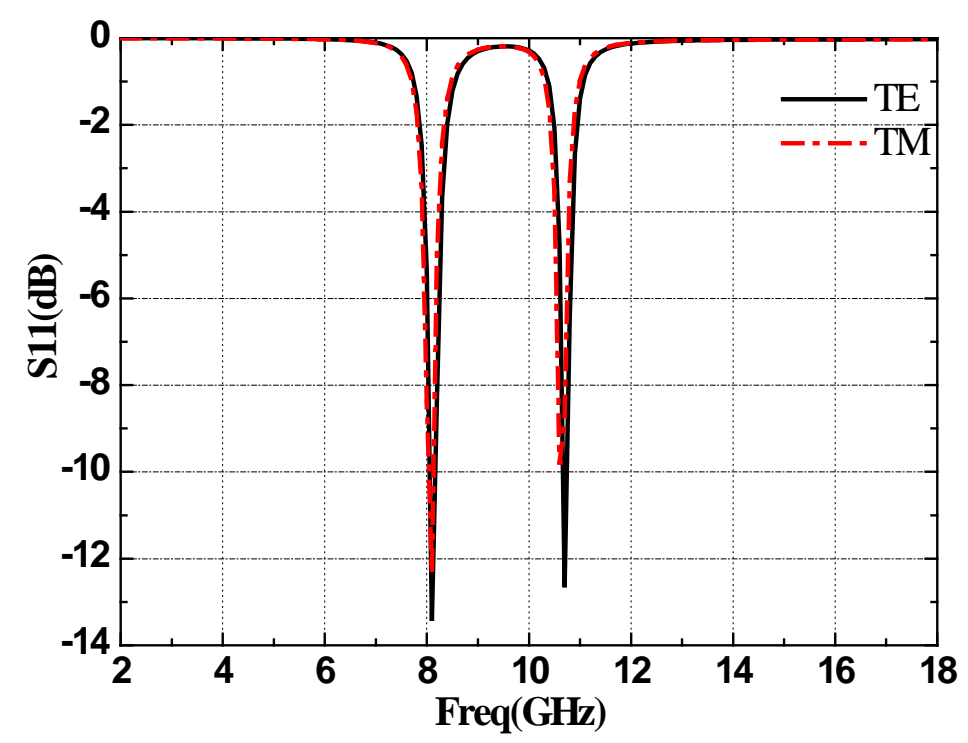

Fig. 5 Reflection coefficient S11 for TE and TM polarization.

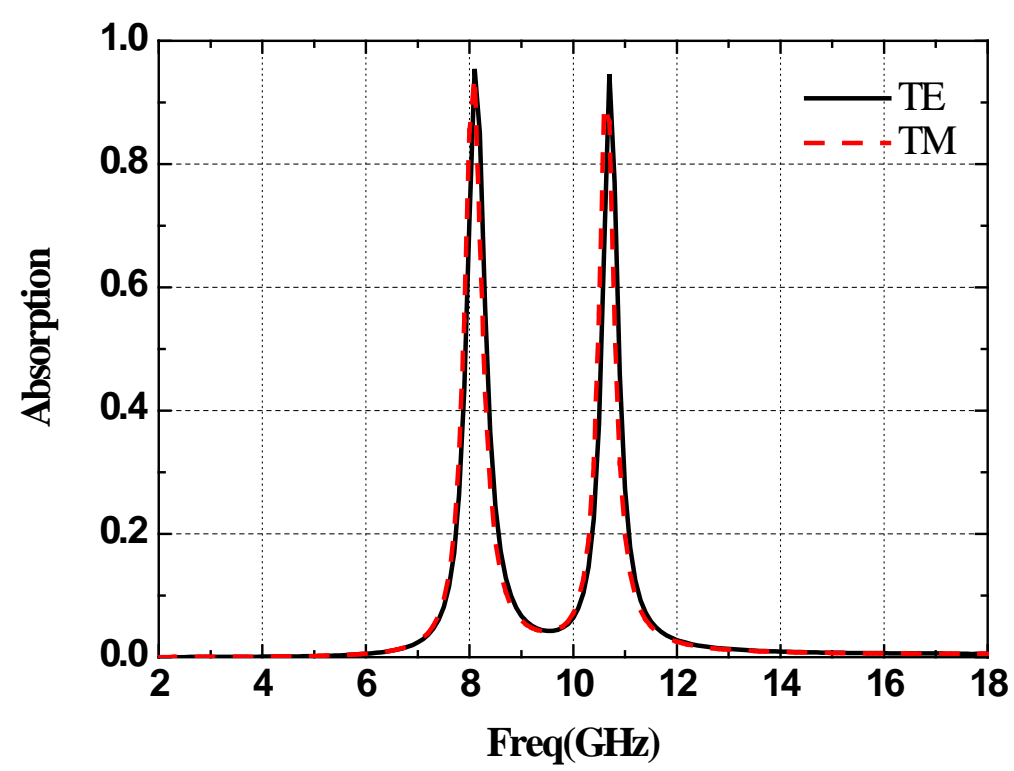

Fig. 6 Absorption rate for TE and TM polarization.

For TE polarization, it can be observed from Fig. 5 that the reflection rate S11 reaches two minimum values at frequencies of $8.1 \mathrm{GHz}$ and $10.7 \mathrm{GHz}$, which correspond to the two absorption peaks with absorption 0.95 and 0.94, respectively (as seen in Fig. 6). Each ring responses for one resonant frequency and is almost independent with each other. Because the structure is symmetrical, it has almost the same response to TM polarization with two absorption peaks at frequencies 8.1GHz and 10.6GHz with absorption 0.94 and 0.90, respectively (as seen in Fig. 6).

\section{Conclusions}

In conclusion, we designed, simulated and analyzed a dual-band and polarization-independent metamaterial absorber with absorption rates of 0.95 and 0.94 at two separated frequencies. The absorber has a simple structure and thus is easy to expand to three or even more band resonant 
absorbers. Therefore, it is a good candidate to design high performance absorbers for potential applications.

\section{References}

[1] T.J. Cui, David R.Smith, R.P. Liu. Metamaterials Theory, Design, and Applications[M]. New York: Springer, 2009:2

[2] N.I.Landy, S. Sajuyigbe, J.J. Mock et al. Perfect Metamaterial Absorber[J]. Phys. Rev. Lett. 2008,100:207402

[3] Veselago V G. The electrodynamics of substances with simultaneously negative values of $\varepsilon$ and $\mu[\mathrm{J}]$. Sov Phys Usp,1968,10:509-514

[4] Pendry J B. Negative refraction makes a perfect lens[J].Physical Review L,2000,85:3966

[5] D.Schurig, J.J. Mock, B.J. Justice et al. Metamaterial Electromagnetic Cloak at Microwave Frequencies[J]. Science, 2006, 314(5810):977-980

[6] Hui Li, L.H. Yuan, Bin Zhou et al. Ultrathin multiband gigahertz metamaterial absorber[J]. Journal of Applied Physics, 2011,110:014909

[7] T. Maier and H. brueckl. Multispectral microbolometers for the midinfrared[J]. Opt. Lett. 2010,35(22):3766-3768 